\title{
Refluxerkrankung - Haben wir es uns zu bequem gemacht?
}

Die gastroösophageale Refluxerkrankung (GERD) ist mit einer Prävalenz von etwa $15 \%$ eine häufige Erkrankung, zahlreiche Studien berichten noch höhere Werte. Das liegt unter anderem an einem hohen overlap zur Dyspepsie. Zusammen kommen beide Symptomenkomplexe auf Prävalenzen von bis zu $30 \%$. Die mögen überschätzt sein, fraglos bleibt aber, dass sich in den Wartezimmern viele Patienten finden, die an GERD-, Dyspepsie-, Beschwerden beider Symptomenkomplexe oder GERDähnlichen, aber nicht typischen Beschwerden leiden.

Die Behandlung der GERD hat sich in den letzten 30 Jahren stark gewandelt. Die Protonenpumpeninhibitoren (PPI) haben es uns ermöglicht, gerade in Bezug auf rein säurebedingte Erkrankungen die Beschwerden unserer Patienten sehr gut zu kontrollieren, auch im Falle von Konsequenzen des sauren Refluxes, wie eine Refluxösophagitis oder Komplikationen wie peptische Stenosen oder Refluxulzerationen. Auch Fragen und Anspruch der GERD/Dyspepsiepatienten haben sich geändert.

Patienten mit klassischem Sodbrennen werden in der Primärversorgung gut versorgt und nur noch selten konsiliarisch beim Gastroenterologen vorgestellt. Hier finden sich nun Patienten bei denen ein PPI nicht oder nicht ausreichend hilfreich ist und sich eben mehr als nur die eine Frage stellt, ob eine erneute Ösophago-Gastro-Duodenoskopie erforderlich ist. Mit welchen weiteren Maßnahmen kann geholfen werden? Die 2014 revidierte DGVS-Leitlinie zur GERD hilft uns in der Diagnostik und Therapie bei vielen dieser Patienten weiter, dennoch bleiben zahlreiche klinische Fragen der Diagnostik und Therapie schwierig.

\section{"Lost patients" und viele offene Fragen}

Die neuen Herausforderungen sind mannigfaltig. Wie sollen wir den pathologischen, nicht sauren Reflux, den wir mit der neuen Technologie der 24-Stunden-Impedanz-pH-Metrie messen können, adäquat und praktikabel behandeln? Wann und wie sollen wir eine PPI-Therapie beenden, besonders vor dem Hintergrund, das schon bei kurzfristiger regelmäßiger PPI-Gabe das Absetzen von einem Säure-Rebound gefolgt wird, der den Patienten mit wiederkehrenden Symptomen in die Praxis zurückbringt, oder wenn dieser trotz Beschwerden aufgrund von Osteoporose- und Demenzpanik nie wieder PPI einnehmen möchte? Wie sollen wir Studien bewerten, die epidemiologisch belegen, dass ein Großteil der GERD-Patienten nicht oder nicht adäquat behandelt ist und die diese inzwischen als verlorene Patienten (lost patients) bezeichnen? Haben wir es uns zu bequem gemacht, indem wir bei Refluxbeschwerden reflexartig einen PPI verschreiben sowie Lebensstil- und Ernährungsempfehlungen geben, bei Versagen der gewählten Maßnahmen aber nicht die gesamte Bandbreite der

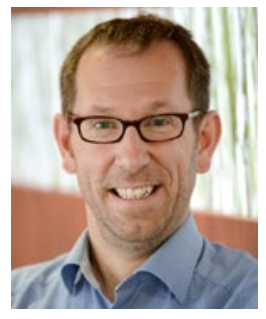

Prof. Dr. med. Martin Storr, MHBA, FRCPC

Zentrum für Endoskopie

Oßwaldstr. 1, 82319 Starnberg

E-Mail: gidoc@gmx.com

verfügbaren Therapieoptionen verwenden, um auf die Individualität der einzelnen Patienten einzugehen? Hat der Erfolg der säuresupressiven PPI-Therapie dazu geführt, dass es jahrelang keine notwendige weitere Forschung im Bereich der GERD gegeben hat, die uns nun ermöglichen würde, die verbliebenen Problemfälle adäquat zu behandeln? Sind genetische Veränderungen im PPI-Metabolismus wie oft angemerkt (rapid/slow metabolizer) unterdiagnostiziert und sollten wir diese möglichen Erklärungsursachen eines PPI-Therapieversagens mehr in den diagnostischen Vordergrund stellen? ... Und wenn ja, mit welcher therapeutischen Konsequenz? Oder sind unsere Patienten eigentlich doch ganz gut versorgt und die wenigen, die noch über Beschwerden klagen nur nicht richtig darüber aufgeklärt, was sie von einer adäquaten Therapie und von ihrem Leben erwarten können?

\section{GERD ist gastroenterologische Kernkompetenz}

Trotz guter Therapieoptionen haben sich also mittlerweile ganz neue Fragen ergeben, auf die wir Antworten benötigen. Hier sei angemerkt, dass der „verlorene“ GERD-Patient vom Gastroenterologen gesucht und gefunden werden sollte, denn GERD-Diagnostik und -Expertise ist Teil der gastroenterologischen Kernkompetenz und sollte gerade bei schwierigen Patienten nicht delegiert werden. GERD-Diagnostik und -Therapie sind intelligent und spannend, sofern die gesamte Klaviatur bespielt wird. Die benötigten Antworten geben uns aktuelle und zukünftige klinische Studien - und es ist sehr erfreulich, dass die Studientätigkeit auf dem Gebiet der GERD zu keinem Zeitpunkt eingeschlafen ist. GERD-Studien finden sich zwar nicht mehr in den Journalen mit dem höchsten Impact-Faktor, aber zuverlässig in Journalen der zweiten Reihe, die jeder von uns aufmerksam verfolgen oder sich alternativ in Medien wie der GASTRO NEWS aktuell über die wichtigsten Neuheiten informieren kann.

Martin Storr 